\title{
RECONSTRUCTION OF 3D VECTOR MODELS OF BUILDINGS BY COMBINATION OF ALS, TLS AND VLS DATA
}

\author{
H. Boulaassal, T. Landes, P. Grussenmeyer \\ Photogrammetry and Geomatics Group, TRIO/LSIIT - UMR 7005 CNRS \\ INSA Strasbourg, Graduate School of Science and Technology \\ 24 Boulevard de la Victoire, 67084 STRASBOURG, France. \\ (hakim.boulaassal, tania.landes, pierre.grussenmeyer)@insa-strasbourg.fr
}

KEY WORDS: Point Cloud, Segmentation, Reconstruction, Building façade, Modelling, Roofs.

\begin{abstract}
:
Airborne Laser Scanning (ALS), Terrestrial Laser Scanning (TLS) and Vehicle based Laser Scanning (VLS) are widely used as data acquisition methods for 3D building modelling. ALS data is often used to generate, among others, roof models. TLS data has proven its effectiveness in the geometric reconstruction of building façades. Although the operating algorithms used in the processing chain of these two kinds of data are quite similar, their combination should be more investigated. This study explores the possibility of combining ALS and TLS data for simultaneously producing 3D building models from bird point of view and pedestrian point of view. The geometric accuracy of roofs and façades models is different due to the acquisition techniques. In order to take these differences into account, the surfaces composing roofs and façades are extracted with the same algorithm of segmentation. Nevertheless the segmentation algorithm must be adapted to the properties of the different point clouds. It is based on the RANSAC algorithm, but has been applied in a sequential way in order to extract all potential planar clusters from airborne and terrestrial datasets. Surfaces are fitted to planar clusters, allowing edge detection and reconstruction of vector polygons. Models resulting from TLS data are obviously more accurate than those generated from ALS data. Therefore, the geometry of the roofs is corrected and adapted according to the geometry of the corresponding façades. Finally, the effects of the differences between raw ALS and TLS data on the results of the modeling process are analyzed. It is shown that such combination could be used to produce reliable 3D building models.
\end{abstract}

\section{INTRODUCTION}

Airborne Laser Scanning (ALS), Terrestrial Laser Scanning (TLS), and Vehicle based Laser Scanning (VLS) are widely used as data acquisition methods for 3D building modelling. The main advantage of the laser scanning technique is that it allows to directly collect georeferenced sets of dense point clouds. Over the last decade, many significant developments in the field of laserscanning acquisition techniques have been performed. Also the laser data processing tools are constantly being improved (Shan and Toth, 2009).

ALS data are widely used in the production of Digital Terrain Models (DTM) and rough building models. The suitability of ALS techniques for 3D object reconstruction has been proven over the last decades. Nevertheless, the automatic generation of DTM as well as 3D building models remains an unresolved issue. This is essentially due to the weakness of information often given by the second echo compared to the first one. Actually, when this one is reliable, it helps to distinguish points belonging to the ground and non-ground surfaces (Tarsha-Kurdi et al., 2006). But in most cases, it is not always separable from the first one (Pfeifer et al., 1999). Currently, researchers rather use fullwaveform ALS data which provides the complete waveform of the backscattered signal produced by the illuminated object. Nevertheless, that kind of data is even rarely acquired and requires knowledge in signal processing techniques.

In all cases, ALS does not directly provide complete 3D models since they do not completely cover building facades. The acquisition angle and also the street narrowness make the façade acquisition difficult. In this case TLS and/or VLS are used. They provide high details point clouds at ground level since the access to the facade is facilitated.

In this context, a combination of ALS and TLS/VLS data for producing 3D building models simultaneously from a bird's point of view and pedestrian's point of view is suggested. This paper is structured as follows. Section 2 gives an overview related to LiDAR data processing. The test site and the used datasets are introduced in section3. Section 4 and 5 explain the processing chain developed for ALS and TLS data modeling respectively. The combination of both is discussed in section 6 .

\section{RELATED WORK}

This section gives an overview of the main processing operations applied on ALS data as well as TLS and VLS data. Concerning the processing of ALS data, it starts with 
the segmentation of acquired points into terrain and offterrain classes. Then the buildings outlines are extracted automatically from the off-terrain class. Globally two approaches are followed for the topic of LiDAR data segmentation and modeling: approaches based on rasterized LiDAR data and approaches based on the raw cloud points. In the first approach, Digital Surface Models (DSM) are produced by interpolation of raw point clouds. Advantage of this approach is that the whole conventional image processing algorithms can easily been applied. Approaches based on the point clouds use generally similarity criteria such as the co-planarity for segmenting the cloud into classes. A combination of both approaches takes advantage of the benefits of both (Tarsha-kurdi et al., 2007).

TLS data has proven its relevance in the geometric reconstruction of building façades more particularly the regular ones. The modeling is carried out either by means of geometric primitives or by meshing techniques. In the first case, extraction of planar clusters from the point cloud is the key of the geometric modeling. Many algorithms have been proposed. They can be arranged in two categories: algorithms based on voting techniques such as RANSAC and Hough transform and algorithms based on merging principles such as surface growing (Boulaassal et al., 2008).

As regards to VLS data, they are rarely studied and processed in the purpose of producing complete 3D building models. The interest focuses on the acquisition of the visible part of facades from moving vehicles. The challenge for mobile mapping is the direct georeferencing of the acquired point cloud. Main difficulty for these systems is the adequate synchronization of the components involved, i.e. GPS/IMU/Laser systems. Vehicle-based laser mobile mapping systems can produce data in the same velocity as that of a vehicle. This high productivity requires processing methods which have to be automatic and robust. Although the operating algorithms used in the processing chain of TLS and VLS data are quite similar, their combination needs to be investigated.

(Haala et al., 2008) have used the point clouds collected by the mobile StreetMapper system to refine the planar façades of an existing 3D building model. The coarse building model has been generated previously from ALS data. In this way, the fine geometric details of building façades like windows, balconies or ornaments has been added using the mobile mapping data. The authors define the overall geometry of buildings in using a polyhedral approximation. More details can be found in (Becker \& Haala, 2007). In terms of acquisition precision, VLS data have also been analyzed by (Alshawa et al., 2009). The same authors have also presented first steps in VLS data segmentation and edge extraction. Work will continue on this path.

\section{DATA DESCRIPTION}

Data used in this paper is composed of ALS, TLS and VLS data covering roofs and facades of the Graduate School of Science and Technology (INSA) of Strasbourg. The school is composed of several buildings, themselves composed of many planar surfaces describing different architectural elements (roofs, windows, planar walls) and characterized by different materials (concrete, glass, stone). Some parts of the studied façades are shown in Figure 1.

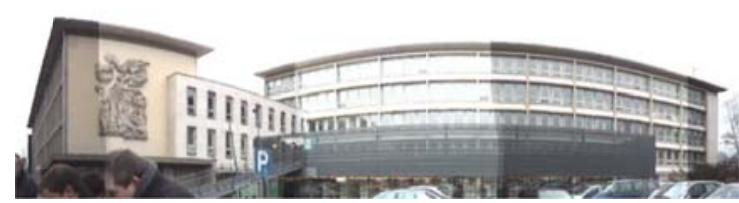

Figure1. Panorama of photographs covering the main facades of INSA Strasbourg

The ALS data used in this project have been acquired in 2004 with the sensor TopScan Optech ALTM 1225. The point cloud covering the INSA is depicted in Figure 2.

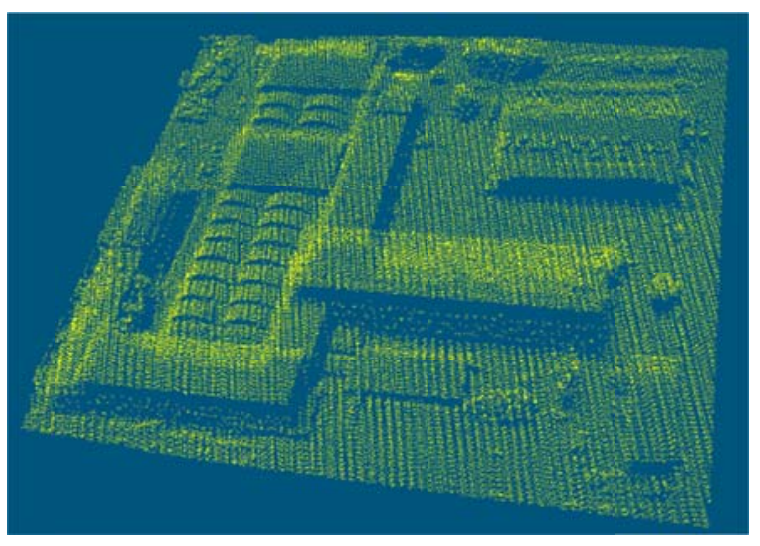

Figure2. Airborne laser scanner data of INSA buildings

The TLS data used in this study is a point cloud acquired by a Trimble GX laser scanner. The VLS data set has been collected by Optech LYNX Mobile Mapper. This system has two rotating laser scanners mounted on the back of a vehicle. The technical specifications of datasets used in this work are summarized in Table 1.

\begin{tabular}{|c|c|c|c|}
\hline \multicolumn{4}{|c|}{ Technical specifications } \\
\hline & ALS & TLS & VLS \\
\hline Sensor & $\begin{array}{c}\text { TopScan } \\
\text { (Optech } \\
\text { ALTM 1225) }\end{array}$ & $\begin{array}{c}\text { Trimble } \\
\text { GX } \\
\text { (Trimble) }\end{array}$ & $\begin{array}{c}\text { LYNX } \\
\text { (Optech) }\end{array}$ \\
\hline $\begin{array}{c}\text { Point } \\
\text { density }\end{array}$ & 2.3 points $/ \mathrm{m}^{2}$ & $\begin{array}{c}185 \\
\text { points } / \mathrm{m}^{2}\end{array}$ & $\begin{array}{c}80 \\
\text { points } / \mathrm{m}^{2}\end{array}$ \\
\hline $\begin{array}{c}\text { Spatial } \\
\text { resolution }\end{array}$ & $\begin{array}{c}0.66 \mathrm{~m} \\
\text { (roof) }\end{array}$ & $\begin{array}{c}0.07 \mathrm{~m} \\
\text { (façade) }\end{array}$ & $\begin{array}{c}0.11 \mathrm{~m} \\
\text { (façade) }\end{array}$ \\
\hline & $\pm 26^{\circ}$ & $-22^{\circ}$ to & $360^{\circ}$ \\
$+38^{\circ}$ & \\
\hline $\begin{array}{c}\text { Points/ } \\
\text { dataset }\end{array}$ & 61618 & 4335496 & 659920 \\
\hline
\end{tabular}

Table 1. Technical characteristics of the ALS, TLS and VLS datasets used in this study.

It is noticed that point densities are averages computed based on 5 samples of each datasiet. The spatial resolutions $(S R)$ are deducted using equation $n^{\circ} 1$ :

$$
S R=\frac{1}{\sqrt{\text { density }}}
$$


ALS and TLS/VLS point clouds are registered in the same coordinate system. The different scans can be merged in the same space, as shown in Figure 3. Nevertheless some shifts between clouds will be noted in section 5 .

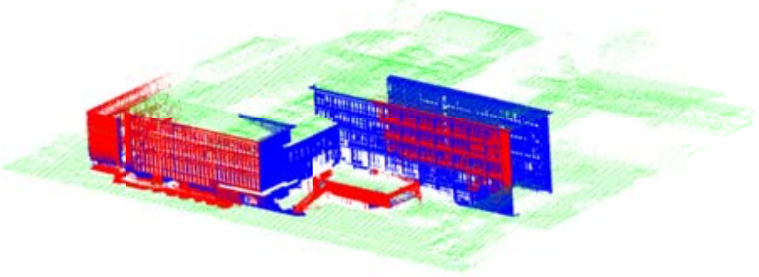

Figure 3. Merging of different datasets: ALS point cloud (green), VLS point cloud (red) and TLS point cloud (blue)

\section{ALS DATA MODELLING}

The processing of ALS data is carried out according the approach developed in our group by (Tarsha-Kurdi et al. 2008). Firstly, the point cloud is transformed into a DSM using a nearest neighbor interpolation. The topological original relationships between points, in the sense of relative height variations between neighboring points, are preserved by this interpolation. Then segmentation is performed on the DSM in order to separate the off-terrain points (building, vegetation, trees) from the ground points. Figure 4 shows the DSM obtained for the point cloud presented in Figure 2. The pseudo-colors are related to the altitudes (in meters).

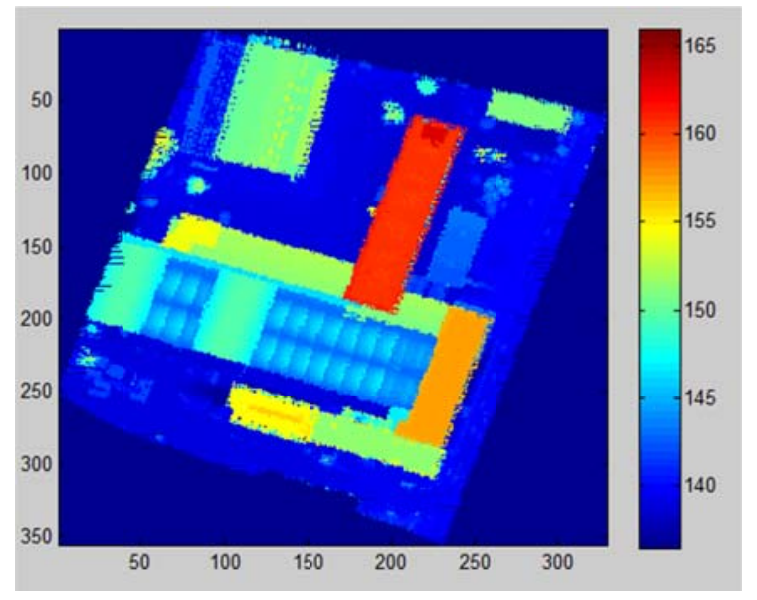

Figure 4. DSM of INSA buildings produced from ALS point cloud

The second stage consists in the discrimination of the offterrain points into vegetation and buildings. The input data of this step is the calculated off-terrain mask containing all off-terrain objects, the DSM and the original point cloud. Figure 5 depicts the results of the buildings mask extraction, for buildings which height is higher than $5 \mathrm{~m}$. Under $5 \mathrm{~m}$, the buildings are not detected.

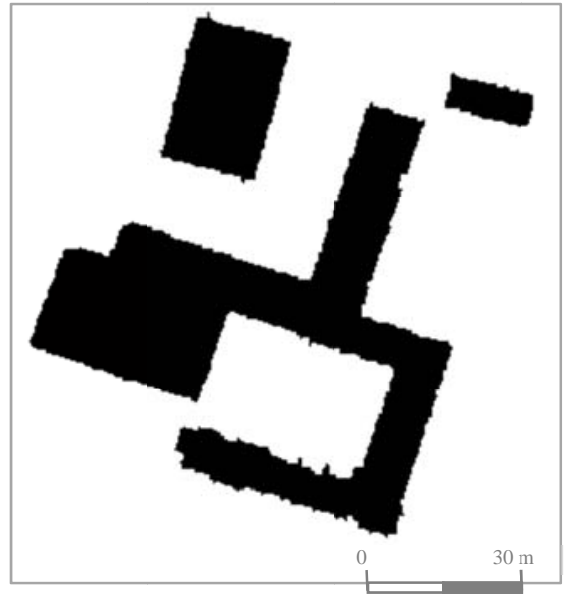

Figure 5. Mask of the INSA buildings under study

Once the building mask is extracted, the process of roof modeling can start. To do this, the outline edges are extracted from the DSM image. Then, they are reconstructed in order to obtain a vector model of the building outlines. Afterwards, planar clusters of roofs are extracted using an improved RANSAC algorithm. These planes provide information about the components of a roof (number, geometry, slope of roof sections) and the height of the building. Based on these parameters, a 3D model of the building can be produced. Façade models are therefore obtained by projection of roof contours onto the ground. Figure 6 presents the reconstructed edges of the INSA buildings outlines, firstly in 2D then in $3 \mathrm{D}$ after extrusion operations.

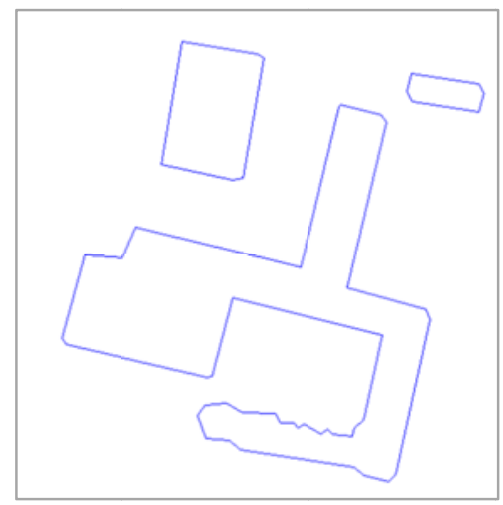

(a)

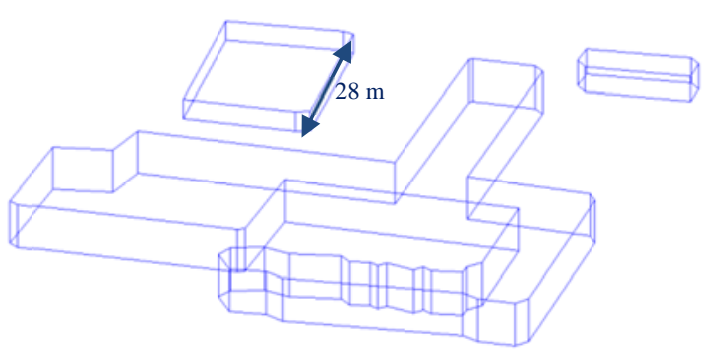

(b)

Figure 6. Buildings models reconstructed from ALS data; (a) 2D outlines of buildings; (b) simplified 3D buildings. 
As it can be seen, the 3D model of the building obtained at this stage, based only on ALS data is simplified. If the roof geometry might be improved (through further processing), it is not possible for the façades. In these models one notes the lack of architectural details such as openings. That's why it is interesting to complete this model by adding the missing elements from ground laser data (VLS and/or TLS).

\section{GROUND DATA MODELLING}

As mentioned above, the ground laser data implies data coming from TLS and/or VLS data. These two kinds of point clouds are complementary. Before modeling the building facades, it is necessary to put together TLS and VLS data. Obviously, the TLS data is considered as absolute reference, because its georeferencing is considered to be more accurate than that of the VLS.

Figure 7 presents the merging of point clouds acquired by static and mobile mapping systems only.



(a)

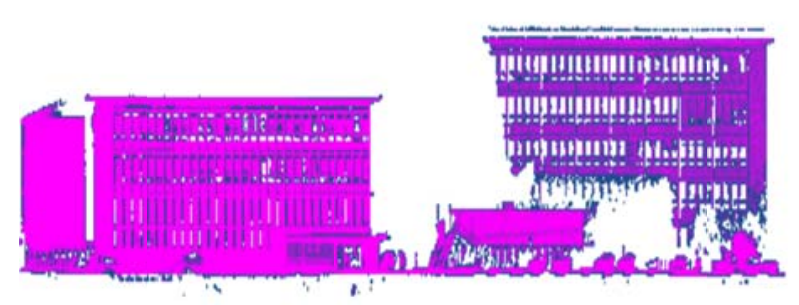

(b)

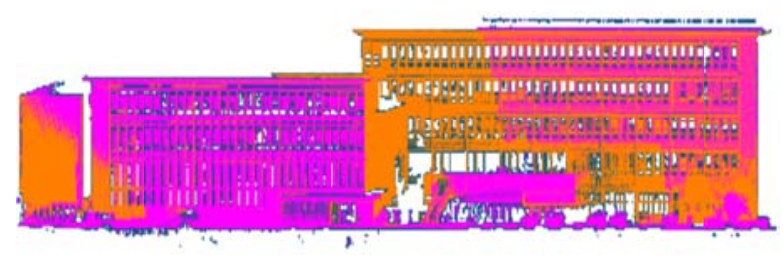

(c)

Figure 7. Merging of TLS and VLS point clouds: (a) TLS point cloud; (b) VLS point cloud; (c) Merged point clouds

The merging has been performed after registration of both point clouds. Since no specific targets have been placed on the façade before data capture, the registration has been performed based on common existing elements. It consists in finding common points or common geometric primitives in both clouds and in performing an ICP (Iterative Closest Points) process to bring them closer.

Then the process of façade modelling can start based on the whole point cloud. The first step consists in sampling data in order to remove redundant points. To do this, an algorithm looking for points whose coordinates are equal at $\pm 1 / 10 \mathrm{~mm}$ has been developed. The number of points is reduced after this sampling.

Relevant features like main façade plane are firstly extracted via a segmentation algorithm. The building studied in this paper is composed mainly by planar surfaces. Therefore, the automatic segmentation algorithm proceeds to the decomposition of the point cloud into a set of planar clusters. The raw point cloud has a thickness coming from the effect of surface roughness, object colors, TLS resolution capacities, registration operations and noise. The planes are thus approximated by planar clusters having some specific thickness. Therefore, a tolerance value describing the authorized thicknesis around a plane has been set. For reaching a reliable and realistic façade model, the RANSAC algorithm has been optimized at several stages. More information about the segmentation algorithm can be found in (Boulaassal et al., 2008).

The segmentation algorithm is intended initially to segment only one façade. The datasets studied in this paper is composed of many facades. Thereby, the point cloud shown in Figure 3 must be decomposed into individual façades. To do this, the building facades obtained from ALS (Figure 6b) are useful. These façades are defined by polygons which vertices are known in 3D coordinates. Each polygon corresponds to at least one façade in the terrestrial point cloud dataset. Points of the point cloud, which distance to the polygon is $\pm 2 \mathrm{~m}$ are considered to belong to the façade. The superimposition of the outlines of façades extracted from ALS data and the point clouids of façades provided by VLS/TLS is depicted in Figure 8.

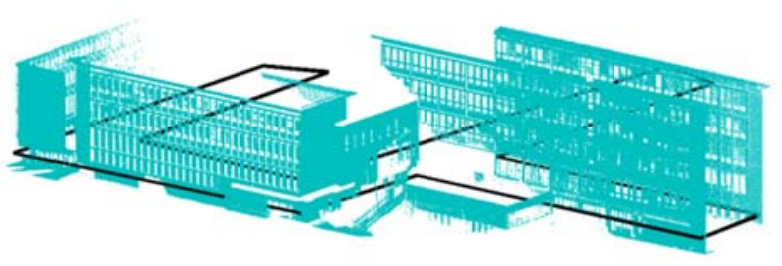

Figure 8. Superimposition of terrestrial data of façades (sky blue) and outlines of façades obtained from ALS data (black contours)

Then each façade is segmented separately using the adapted RANSAC algorithm mentioned previously. Segmentation results obtained over all facades are presented in Figure 9. Since the raw cloud of a planar surface has a thickness as mentioned in section 5, a tolerance value describing the authorized thickness around a plane is set. In our case, this value has been set to $2.5 \mathrm{~cm}$ for TLS data and $4 \mathrm{~cm}$ for the merged TLS and VLS data. 


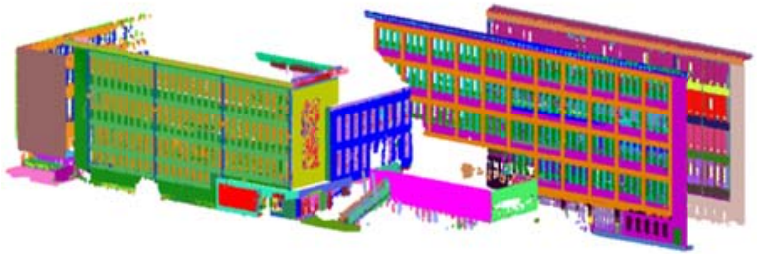

Figure 9. Segmentation results obtained on a VLS/TLS merged point cloud. Each color depicts one planar architectural element.

Afterwards, based on the segmentation results, the automatic extraction of edges can be achieved for each architectural element. An automatic extraction algorithm of facade contours has been developed to extract the laser points describing the contour of openings or edges between walls. It is based on an automatic analysis of a Delaunay triangulation applied on the segmented point cloud. Based on these results, the geometric reconstruction algorithm produces a vector model of the façade. The contours are decomposed into straight and curved edges, based on the criterion of point's collinearity. Then, the geometric reconstruction of edges is performed based on the study of their geometric characteristics, as well as their topological relationships. The vector model of each planar cluster and of the edges that describe the façade elements is available. More information about this step of reconstruction can be found in Boulaassal et al.(2010). Figure 10 shows the vector model of the multiple facades composing the INSA buildings.



Figure 10. Vector models of segmented building facades shown in figure 9.

At this stage, it is interesting to combine these results to those obtained from ALS processing as it is explained in the next section.

\section{COMBINATION OF AIRBORNE AND GROUND DATA MODELLING}

As shown in section 4, ALS data are used to generate more or less detailed roof models and rough models of facades. Figure 11 shows the vector models of façades depicted in Figure 10, combined with the roofs segments extracted from ALS data. It should be noticed that, for ALS data, the tolerance value set for planar cluster definition was $40 \mathrm{~cm}$.

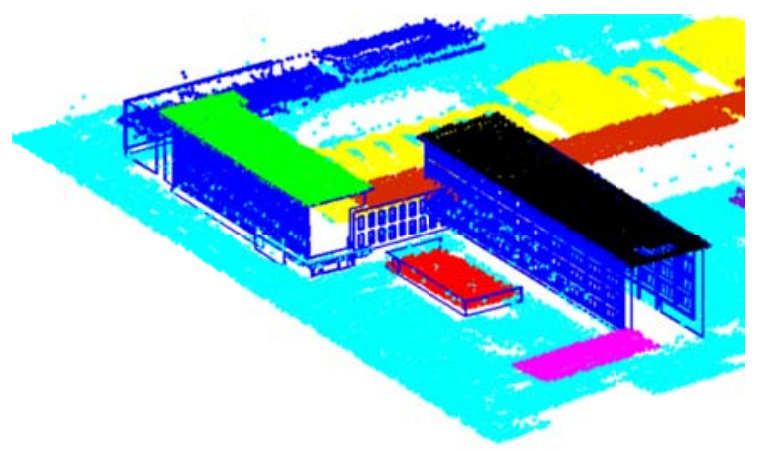

Figure 11. Combination of vector models of INSA façades and segmented ALS point cloud.

The planar clusters of the roofs are modeled in the same way that terrestrial ones. Firstly, point edges of each plane are extracted using the same algorithm developed initially for TLS data (Boulaassal et al., 2010). Figure 12 depicts the result of the combination.

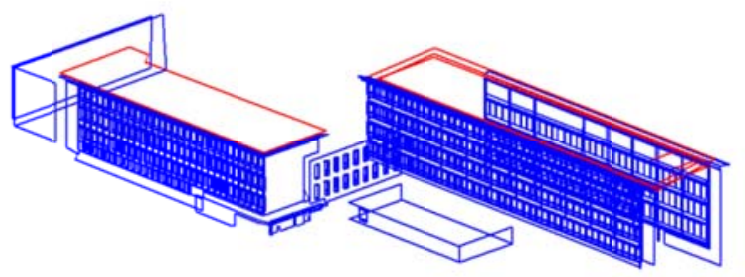

Figure 12. Result of the combination of vector models obtained from airborne (red color) and terrestrial data (blue)

The effect of differences in data quality can be remarked in the resulting model. As expected, polygons of roofs produced from airborne data do not perfectly superimpose with those produced from terrestrial data. Shifts of about 75 $\mathrm{cm}$ are observed between ALS and TLS polygons more particularly along the $\mathrm{Z}$ axis direction, as shown in Figure 13.

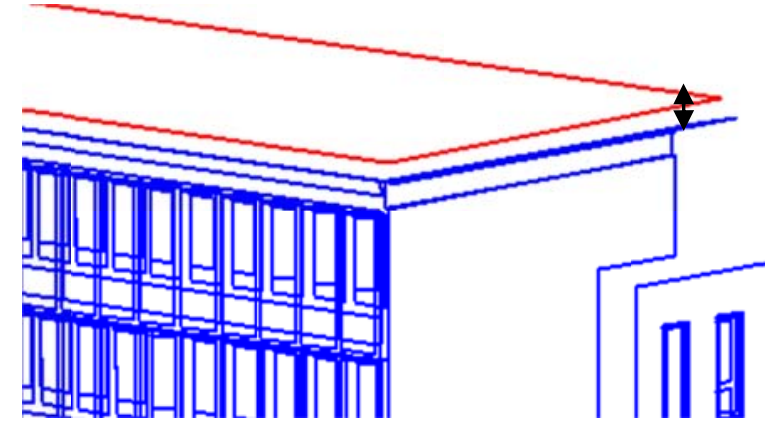

Figure 13. Shift between vector models. The red plane lies $75 \mathrm{~cm}$ over the right position

Such shift can be explained by differences in terms of accuracies of raw data, and in terms of modeling parameters. For instance, in the segmentation process of VLS/TLS data, only $2 \mathrm{~cm}$ are used as threshold value around planar clusters against $40 \mathrm{~cm}$ in the segmentation process of ALS data. The terrestrial vector model should be used as reference model in order to correct the airborne georeferencing. 


\section{CONCLUSION}

The point cloud acquired by an airborne LiDAR system and combined to VLS and TLS point clouds represent a model in itself, since the set of points provides a primary description of the buildings geometry. However, integration and management of these raw data in databases is problematic because of the huge amount of points. The 3D geometric modelling seems to be a good solution to this issue. Our approach enables the transformation of a model composed of points to a model composed of a small number of geometric shapes. In this form, the model is a starting point for other types of information, such as semantic and architectural information.

Properties of ALS, TLS and VLS data are different since they are collected in different ways. However, these differences can be exceeded by taking benefit from each one of them. TLS data must be used as geometric reference because the georeferencing and generally the inherent geometry of the clouds are more accurate than that of ALS data. Therefore the vector model of the roofs obtained from ALS data processing can be improved. ALS data is useful for a coarse modelling of façades which helps in the localization of individual façades and determining the hold of building. We can state that the airborne and ground LiDAR data are complementary if the georeferencing is controlled and corrected before processing. In that case, complete and reliable $3 \mathrm{D}$ models of buildings can be obtained.

In the future, more efforts will be achieved in the statistical study of combination of datasets coming from several laser scanning systems. The contribution of each processing steps in terms of processing time and disk space gain will also be studied.

\section{REFERENCES}

Alshawa, M., Grussenmeyer, P., Smigiel, E., 2009. A lowcost TLS based land mobile mapping system assisted by photogrammetry. Bol. Ciênc. Geod., v. 15, n. 5 - Special Issue on Mobile Mapping Technology, p. 743-761, 2009. ISSN: 1982-2170.

Becker, S. and Haala, N., 2007. Combined feature extraction for façade reconstruction. Proceedings of ISPRS Workshop on Laser Scanning 2007 and SilviLaser 2007, Espoo, September 12-14, 2007, Finland.

Boulaassal, H., Chevrier, C., Landes, T., 2010. From Laser Data to Parametric Models: Towards an Automatic Method for Building Façade Modelling. EuroMed 2010, Lecture Notes in Computer Science. Springer-Verlag Berlin Heidelberg 2010. 6436 Pages. pp. 42-55.

Boulaassal, H., Landes, T., Grussenmeyer, P., 2008. Automatic extraction of planar clusters and their contours on building façades recorded by terrestrial laser scanner, In: Proceedings of the 14th International Conference on Virtual Systems and Multimedia: VSMM 2008, Dedicated to Digital Heritage, Limassol, Cyprus. pp.8-15.

Haala, N., Peter, M., Cefalu, A., Kremer, J., 2008. Mobile lidar mapping for urban data capture. VSMM 2008 - Conference on Virtual Systems and MultiMedia Dedicated to Digital Heritage. Limassol, Cyprus, October 20th - 25th, 2008. Project paper. pp. 101-106
Pfeifer, N., Reiter, T., Briese, C., Rieger, W., 1999. Interpolation of high quality ground models from laser scanner data in forested areas. Joint Workshop of the ISPRS working groups III/5 and III/2 , La Jolla, CA, USA, Nov. 9 - 11 1999.

Shan, J. and Toth, C.K., 2008. Topographic laser ranging and scanning - Principles and Processing. Taylor and Francis Group. 590 pp.

Tarsha-Kurdi, F., Landes, T., Grussenmeyer, P., 2008. Extended RANSAC algorithm for automatic detection of building roof planes from Lidar data. The Photogrammetric Journal of Finland. Vol. 21, n²1, 2008, pp.97-109.

Tarsha-Kurdi, F., Landes, T., Grussenmeyer, P., 2007. Joint combination of point cloud and DSM for 3D building reconstruction using airborne laser scanner data. 4th IEEE GRSS / WG III/2+5, VIII/1, VII/4 Joint Workshop on Remote Sensing \& Data Fusion over Urban Areas and 6th International Symposium on Remote Sensing of Urban Areas. 11-13 April, Télécom Paris. 1-4244-07125/07/\$20.00 C2007 IEEE, 7 pp.

Tarsha-Kurdi, F., Landes, T., Grussenmeyer, P. and Smigiel, E., 2006. New approach for automatic detection of buildings in airborne laser scanner data using first echo only. IAPRS Vol. 36, part. 3, pp. 25-30. 